\title{
Supercurrent and its Quantum Statistical Properties in Mesoscopic Josephson Junction in the Presence of Nonclassical Light Fields
}

\author{
Le-Man Kuang††, Yiwen Wang $\dagger$ and Mo-Lin Ge $\dagger$ \\ $\dagger$ Theoretical Physics Division, Nankai Institute of Mathematics, \\ Tianjin, 300071, People's Republic of China \\ $\ddagger$ Department of Physics and Institute of Physics, Hunan Normal University, \\ Hunan 410006, People's Republic of China
}

\begin{abstract}
In this paper, we study the supercurrent in a mesoscopic Josephson junction (MJJ) and its quantum statistical properties in the presence of nonclassical light fields. We investigate in detail the influence of external nonclassical light fields on current-voltage step structures of the MJJ. We also study in detail quantum statistical properties of the supercurrent when the external quantum electromagnetic fields are even and odd coherent-state light fields. It is shown that the supercurrent in the MJJ exhibits both squeezing effect and quantum coherences. It is demonstrated that the MJJ can feel the difference not only between classical light fields and nonclassical light fields but also between different nonclassical light fields.
\end{abstract}




\section{Introduction}

In last few years considerable attention has been devoted to mesoscopic Josephson junctions (MJJ) [1-6] due to the prediction of new quantum effects $[1,7-10]$ in these small-capacitance junctions. Much work concentrated on the macroscopic quantum tunnelling of the phase of current-biased junctions and the influence of dissipation on them [11-15]. It is well known that the effect of classical electromagnetic fields(EMF) on macroscopic Josephson junctions has been well understood. The Shapiro steps[16] are the result that the external static EMF and time-varying EMF work together on Josephson junctions. It is natural to consider the effect of quantum EMF on Josephson junctions. In particular, it is an interesting topic to study the effect of quantum EMF on the MJJ since it can respond to the quantum nature of the external EMF.

On the other hand, nonclassical light fields [17] have been studied extensively in the recent years and produced experimentally. These studies emphasize nonclassical effects of light fields such as squeezing effect and sub-Poissonian distribution to reflect the quantum nature of light which can not be understood classically. Even and odd coherent states(CS) 18, 19 are two types of important nonclassical states of light and have wide applications in physics [21-24]. Although even and odd CS can be decomposed into superpositions of the Glauber CS, their properties are quite different from those of the Glauber CS since the former are nonclassical states while the latter are classical ones 25]. On the other hand, even CS and odd CS exhibit significantly different nonclassical characteristic separately [19, 26]. Even CS exhibits quadrature squeezing but does not sub-Poissonian photon statistics while odd CS exhibits sub-Poissonian but does not quadrature squeezing.

More recently, Vourdas [27] has studied the effect of external quantum EMF on the MJJ by the use of a circular superconducting device with a MJJ in the presence of quantum EMF. He has investigated the step structures of the current and voltage of 
the MJJ. It has been shown that these step structures are similar to Shapiro steps in macroscopic Josephson junctions, but their details depend on the nature of quantum EMF.

The aim of this paper is to study systematically properties of the supercurrent through the MJJ in the presence of nonclassical EMF by taking even and odd CS as an example of nonclassical EMF and discuss effects of the external nonclassical EMF on these properties concretely. We shall study the current-voltage step structures of the MJJ and quantum statistical properties of the supercurrent. This paper is organized as follows: in Sec.2 the current-voltage step structures (CVSS) of supercurrents in the MJJ and the effect of nonclassical EMF on them are investigated. Sec.3 and Sec.4 are devoted to study quantum statistical properties of the supercurrent. Squeezing properties and quantum coherences are investigated in detail for even and odd CS EMF. The results are summarized and conclusions are drawn in the last section.

\section{CVSS of the MJJ in the presence of even and odd coherent-state EMF}

Consider a MJJ with a capacitance $C$ in a circular superconducting device [27]. The MJJ is described by the following Hamiltonian $(\hbar=c=1)$

$$
H=\frac{(q+Q)^{2}}{2 C}+E_{J}(1-\cos \theta)
$$

where the first term is the charging energy, and the second one is the Josephson coupling energy; $q$ is the charge on one of the electrodes of the MJJ, and $Q$ is the external charge; $\theta$ is the phase difference of the superconducting order parameters in the two electrodes; and $E_{J}$ is the Josephson coupling constant.

Capacitance $C$ of conventional Josephson junctions and the temperatures where we usually operate allow us to neglect the charging energy in (11) and to forget the quantum mechanical origin of the Josephson effect. In stead, we view the Josephson junction as 
a classical object whose state is characterized by a sharp value of the phase difference across the junction. However, In the case of the MJJ, the classical descriptions is no longer sufficient, and the charging energy is not negligible. We have to describe the MJJ in a fully quantum mechanical way. In this case, the charge $q$ and the phase difference $\theta$ are a pair of quantum mechanical conjugation variables with $\hat{q}=-i 2 e \partial_{\theta}$, they satisfy the canonical commutation relation:

$$
[\hat{\theta}, \hat{q}]=i 2 e
$$

The Hamiltonian (11) is then rewritten as

$$
H=-\frac{1}{2} E_{c}\left(\partial_{\theta}+i \frac{Q}{2 e}\right)^{2}+E_{J}(1-\cos \hat{\theta})
$$

where the Josephson coupling energy related to the critical current $I_{c r}$ and the charging energy associated with the Cooper-pair charge $2 e$ are given by, respectively,

$$
E_{J}=\frac{I_{c r}}{2 e}, \quad E_{c}=\frac{(2 e)^{2}}{C^{2}}
$$

Starting with the Hamiltonian (3), one can find that the Josephson-current operator $\hat{I}$ in the Heisenberg picture takes the form:

$$
\hat{I}(t)=I_{c r} \sin \hat{\theta}(t)
$$

As is known, the Shapiro steps on macroscopic Josephson junctions is the result which the classical static electromotive force work together with the classical timevarying electromotive force. In the present case, we still impose the classical static electromotive force $V_{0}$ but the quantum time-varying electromotive force on the MJJ through applying a classical magnetic flux $\phi=V_{0} t$ and a ac quantum single-mode magnetic field of angular frequency $\omega_{1}$, respectively. Then, the external voltage induced by the quantum magnetic field in the Heisenberg picture can be written as

$$
V_{e x}(t)=\frac{i \omega_{1}}{\sqrt{2}}\left(e^{i \omega_{1} t} a^{+}-e^{-i \omega_{1} t} a\right)
$$


where $a$ and $a^{+}$are the annihilation and creation operators of the quantum EMF. we consider the case $\omega_{1} \gg \sqrt{E_{c} E_{J}}$ where $\sqrt{E_{c} E_{J}}$ is the angular frequency of the MJJ when it is approximated as a harmonic oscillator. In this case the Hamiltonian of the MJJ itself produces an evolution which is much slower than the Hamiltonian describing the effect from external fields, and the phase evolves approximately in the form [27]:

$$
e^{i \hat{\theta}(t)}=e^{i \omega_{0} t} D\left(\xi e^{i \omega_{1} t}\right)
$$

where $\omega_{0}=2 e V_{0}, \xi=\sqrt{2} e$ ( $e$ is the charge of the electron), and $D(Z)$ is the displacement operator defined by

$$
D(Z)=e^{Z a^{+}-Z^{*} a}
$$

Then the expectation value of the Josephson current $I$ can be given by tracing of the current operator $\hat{I}$ over the density operator of the external quantum EMF,

$$
I=I_{c r} \operatorname{Im}\left\{e^{i \omega_{0} t} \operatorname{Tr}\left[\rho D\left(\xi e^{i \omega_{1} t}\right)\right]\right\}
$$

It is interesting to note that the density operator $\rho$ of the external quantum EMF controls the operation of the MJJ in the sense that the Josephson current and also all the other quantities, one might wish to calculate, depend on the density operator $\rho$. So that we can change the state in which the junction operates through changing the density matrix of the external quantum EMF. This gives a way to realize the controlling of the Josephson tunnelling.

We now consider the current-voltage step structures of the MJJ when the external quantum EMF are in even and odd coherent states, respectively, and investigate the effect of quantum nature of the external quantum EMF on the step structures.

The number-state representations of even and odd CS are defined by

$$
|Z\rangle_{e}=N_{e}(|Z|) \sum_{n=0}^{\infty} \frac{Z^{2 n}}{\sqrt{(2 n) !}}|2 n\rangle, \quad N_{e}(|Z|)=\cosh ^{-\frac{1}{2}}\left(|Z|^{2}\right)
$$




$$
|Z\rangle_{o}=N_{o}(|Z|) \sum_{n=0}^{\infty} \frac{Z^{2 n+1}}{\sqrt{(2 n+1) !}}|2 n+1\rangle, \quad N_{o}(|Z|)=\sinh ^{-\frac{1}{2}}\left(|Z|^{2}\right)
$$

which can be decomposed into superpositions of Glauber CS $|Z\rangle$ and $|-Z\rangle$ in the form

$$
\begin{aligned}
|Z\rangle_{e}=A_{+}^{\frac{1}{2}}(|Z|)(|Z\rangle+|-Z\rangle), & A_{+}^{-1}(|Z|)=2\left(1+e^{-2|Z|^{2}}\right), \\
|Z\rangle_{o}=A_{-}^{\frac{1}{2}}(|Z|)(|Z\rangle-|-Z\rangle), & A_{-}^{-1}(|Z|)=2\left(1-e^{-2|Z|^{2}}\right) .
\end{aligned}
$$

It should be noted that although even and odd CS have the above simple decompositions of Glauber CS, they have quite different quantum nature since even and odd CS light fields are nonclassical light fields while the Glauber CS light field is the classical one [19, 25].

To get the Josephson current, one needs the following matrix elements of the displacement operator in the CS representation

$$
\begin{aligned}
& D_{ \pm Z, \pm Z}\left(\xi e^{i \omega_{1} t}\right) \equiv\left\langle \pm Z\left|D\left(\xi e^{i \omega_{1} t}\right)\right| \pm Z\right\rangle=\exp \left(-\frac{1}{2} \xi^{2} \pm i 2 u\right) \\
& D_{ \pm Z, \mp Z}\left(\xi e^{i \omega_{1} t}\right) \equiv\left\langle \pm Z\left|D\left(\xi e^{i \omega_{1} t}\right)\right| \mp Z\right\rangle=\exp \left(-\frac{1}{2} \xi^{2}-2|Z|^{2} \pm 2 v\right)
\end{aligned}
$$

where

$$
u=\xi|Z| \sin \left(\omega_{1} t-\varphi\right), \quad v=\xi|Z| \cos \left(\omega_{1} t-\varphi\right)
$$

Here we have taken $Z=|Z| e^{i \varphi}$.

Making use of Eqs. (14) and (15), from the expressions (77) and (9) one can obtain the expectation values of the phase-difference operator in the even and odd CS with the results

$$
\begin{aligned}
& { }_{e}\left\langle Z\left|e^{i \theta(t)}\right| Z\right\rangle_{e}=2 A_{+}(|Z|) e^{i \omega_{0} t} e^{-\frac{1}{2} \xi^{2}}\left(\cos 2 u+e^{-2|Z|^{2}} \cosh 2 v\right), \\
& { }_{o}\left\langle Z\left|e^{i \theta(t)}\right| Z\right\rangle_{o}=2 A_{-}(|Z|) e^{i \omega_{0} t} e^{-\frac{1}{2} \xi^{2}}\left(\cos 2 u-e^{-2|Z|^{2}} \cosh 2 v\right)
\end{aligned}
$$

and the Josephson current in the presence of even and odd coherent-state EMF,

$$
\begin{aligned}
& I_{e}=2 I_{c r} A_{+}(|Z|) e^{-\frac{1}{2} \xi^{2}}\left(\cos 2 u+e^{-2|Z|^{2}} \cosh 2 v\right) \sin \omega_{0} t \\
& I_{o}=2 I_{c r} A_{-}(|Z|) e^{-\frac{1}{2} \xi^{2}}\left(\cos 2 u-e^{-2|Z|^{2}} \cosh 2 v\right) \sin \omega_{0} t
\end{aligned}
$$


In order to see the CVSS of the above currents, we expand the rhs of Eqs. (19) and (20) as follows:

$$
\begin{aligned}
I_{e}= & 2 I_{c r} A_{+}(|Z|) e^{-\frac{1}{2} \xi^{2}} \sum_{n=-\infty}^{\infty}\left[J_{2 n}(2 \xi|Z|)\right. \\
& \left.+e^{-2|Z|^{2}} I_{2 n}(2 \xi|Z|)\right] \sin \left[\left(2 n \omega_{1}+\omega_{0}\right) t-2 n \varphi\right], \\
I_{o}= & 2 I_{c r} A_{-}(|Z|) e^{-\frac{1}{2} \xi^{2}} \sum_{n=-\infty}^{\infty}\left[J_{2 n}(2 \xi|Z|)\right. \\
& \left.-e^{-2|Z|^{2}} I_{2 n}(2 \xi|Z|)\right] \sin \left[\left(2 n \omega_{1}+\omega_{0}\right) t-2 n \varphi\right]
\end{aligned}
$$

where $J_{n}(x)$ and $I_{n}(x)$ are Bessel and modified Bessel functions, respectively, and we have used the formulae:

$$
e^{i x \sin \theta}=\sum_{n=-\infty}^{\infty} J_{n}(x) e^{i n \theta}, \quad e^{x \cos \theta}=\sum_{n=-\infty}^{\infty} I_{n}(x) e^{i n \theta} .
$$

The dc components of the Josephson currents can be obtained through taking the time meanvalues of the expressions (21) and (22) in the interval $(0, t)$. It can be seen from Eqs. (21) and (22) that for even CS and also for odd CS when the de voltage $V_{0}$ obeys the following condition

$$
2 n \omega_{1}-\omega_{0}=0, \text { i.e., } V_{0}=\frac{n \omega_{1}}{e}
$$

we get Shapiro dc currents similar to those in macroscopic junctions. Their expressions take the form

$$
\begin{aligned}
& I_{e}^{d c}=2 I_{c r} A_{+}(|Z|) e^{-\frac{1}{2} \xi^{2}}\left[J_{-2 n}(2 \xi|Z|)+e^{-2|Z|^{2}} I_{-2 n}(2 \xi|Z|)\right] \sin (2 n \varphi), \\
& I_{o}^{d c}=2 I_{c r} A_{-}(|Z|) e^{-\frac{1}{2} \xi^{2}}\left[J_{-2 n}(2 \xi|Z|)-e^{-2|Z|^{2}} I_{-2 n}(2 \xi|Z|)\right] \sin (2 n \varphi) .
\end{aligned}
$$

It can be seen from Eqs. (24), (25) and (26) that even CS and odd CS have similar CVSS. Especially, they share the same voltage step structures when $\varphi=\frac{k \pi}{n}(n \neq$ $0, k=0, \pm 1, \pm 2, \ldots)$, while the dc components vanish, i.e., $I_{e}^{d c}=I_{o}^{d c}=0$; and when $|Z| \gg 1$, we find that even CS and odd CS have almost equal de components, that 
is $I_{e}^{d c} \doteq I_{o}^{d c} \doteq I_{c r} e^{-\frac{1}{2} \xi^{2}} J_{-2 n}(2 \xi|Z|) \sin (2 n \varphi)$. Thus, when $|Z| \gg 1$, even and odd CS have the same CVSS. Nevertheless, when $|Z| \ll 1$, from Eqs. (25) and (26) we have $I_{o}^{d c} \doteq 0$ and $I_{e}^{d c} \doteq \frac{1}{2} I_{c r} e^{-\frac{1}{2} \xi^{2}}\left[J_{-2 n}(2 \xi|Z|)+I_{-2 n}(2 \xi|Z|)\right] \sin (2 n \varphi)$ which indicate that when $|Z| \ll 1$ even and odd CS have quite different CVSS. Therefore, the MJJ can feel the difference between different external nonclassical EMF.

On the other hand, the CVSS of even and odd CS are also different from that of the Glauber CS [27]. The voltage jumps (24) in the former are double in size in comparison with the voltage jumps in the latter. This means that the electrostatic energy that the Cooper pair loses during tunnelling in the case of even and odd CS is twice of that in the Glauber CS case. As concerns the dc components of the Josephson currents, the dc components for even and odd CS have only one direction for all steps for a given $Z$ while for the Glauber CS the de components have two directions which correspond to $n$ being even and odd, respectively. Taking into account the results in Ref. [27], we can find that the Glauber CS, CS with thermal noise, and CS with partially randomized phase have the same CVSS, while even and odd CS, squeezed vacuum states exhibit the same CVSS. This reflects the fact that the former three types of states are classical states while the latter three ones are nonclassical states. Therefore, we can conclude that the MJJ can also feel the difference between the external nonclassical EMF and classical ones.

It is worthwhile mentioning that the existence of the CVSS of the MJJ depends strongly on the form of the density matrix describing the external quantum EMF. Those states which off-diagonal elements of their density matrices in the number-state representation are nonzero, such as the Glauber CS, even and odd CS, squeezed vacuum states, exhibit the CVSS of the MJJ, while for those states which off-diagonal elements of the density matrices are zero, such as CS with randomized phase, thermal states and number states, there do not exist the CVSS. This indicates that the existence of the 
CVSS of the MJJ are determined by the external dc voltage $V_{0}$ and the off-diagonal elements of the density matrices of the external quantum EMF. In other words, the step structures depends on the dc voltage $V_{0}$ and the quantum interference among the number states of the external quantum EMF. In this sense, the step structures reflect to some extend quantum coherences of the external quantum EMF.

\section{$3 \quad$ Squeezing properties of supercurrents in the MJJ in the presence of quantum EMF}

Squeezing is one of the most important concepts in quantum optics [25, 28]. It has potential applications in low-noise optical communications and high-precision interferometic measurements. It is of importance to study squeezing behaviors of phase in quantum mechanics [29, 30]. As is known, the supercurrent in a Josephson junction is a quantum-phase-correlation phenomenon. So it is interesting to study squeezing properties of the supercurrent. In this section, we discuss squeezing of the supercurrent in the MJJ in the presence of quantum EMF.

Consider two noncommuting Hermitian operators $\hat{A}$ and $\hat{B}$ acting on the state space of the external quantum EMF. The variances (fluctuations) of these operators $\left\langle(\Delta \hat{A})^{2}\right\rangle=\left\langle\hat{A}^{2}\right\rangle-\langle\hat{A}\rangle^{2}$ and $\left\langle(\Delta \hat{B})^{2}\right\rangle=\left\langle\hat{B}^{2}\right\rangle-\langle\hat{B}\rangle^{2}$ obey the Heisenberg uncertainty relation

$$
\left\langle(\Delta \hat{A})^{2}\right\rangle\left\langle(\Delta \hat{B})^{2}\right\rangle \geq \frac{1}{4}|\langle[\hat{A}, \hat{B}]\rangle|^{2}
$$

which is fundamentally different from that of the position and momentum operators of a quantum mechanical particle $\left\langle(\Delta \hat{x})^{2}\right\rangle\left\langle(\Delta \hat{p})^{2}\right\rangle \geq \frac{1}{4}$ since the commutator $[\hat{A}, \hat{B}]$ on the rhs of equation(27) is not a $C$ number but an operator which makes the rhs of the uncertainty relation(27) to be state-dependent. Uncertainty relation with a state-dependent rhs is well known from earlier studies of the atomic coherent states

[31, 32, 33]. Following Wódkiewicz and Eberly [33] we shall say that the variances of 
the operators $\hat{A}$ and $\hat{B}$ are squeezed if

$$
\left\langle(\Delta \hat{A})^{2}\right\rangle<\frac{1}{2}|\langle[\hat{A}, \hat{B}]\rangle| \text { or }\left\langle(\Delta \hat{B})^{2}\right\rangle<\frac{1}{2}|\langle[\hat{A}, \hat{B}]\rangle|
$$

In order to measure the degree of squeezing of these operators, we introduce two squeezing parameters $S_{A}$ and $S_{B}$ defined by

$$
S_{A}=\frac{\left\langle(\Delta \hat{A})^{2}\right\rangle-\frac{1}{2}|\langle[\hat{A}, \hat{B}]\rangle|}{\frac{1}{2}|\langle[\hat{A}, \hat{B}]\rangle|}, \quad S_{B}=\frac{\left\langle(\Delta \hat{B})^{2}\right\rangle-\frac{1}{2}|\langle[\hat{A}, \hat{B}]\rangle|}{\frac{1}{2}|\langle[\hat{A}, \hat{B}]\rangle|}
$$

The squeezing condition now takes the simple form:

$$
S_{A}<0 \text { or } S_{B}<0
$$

The maximum $(100 \%)$ squeezing of variance $\left\langle(\Delta \hat{X})^{2}\right\rangle$ corresponds to $S_{X}=-1(\hat{X}=$ $\hat{A}$ or $\hat{B})$.

For the present case of the MJJ in the presence of quantum EMF, from the canonical commutation relation of the charge on the electrodes and the phase difference operator (2), we can obtain the commutation relation between the supercurrent operator and the charge operator,

$$
[\hat{I}, \hat{q}]=-i 2 e I_{\mathrm{cr}} \cos \theta
$$

Then, the degree of the squeezing of the Josephson current is given by

$$
S_{I}=\frac{\left\langle(\Delta \sin \hat{\theta})^{2}\right\rangle-e|\langle\cos \hat{\theta}\rangle|}{e|\langle\cos \hat{\theta}\rangle|}
$$

For simplicity we have taken $I_{\mathrm{cr}}$ as the unit of measuring the supercurrent.

For even and odd CS, it is easy to get

$$
\begin{aligned}
{ }_{e}\left\langle Z\left|e^{i \hat{\theta}(t)}\right| Z\right\rangle_{e}= & e^{i \omega_{0} t}\left[D_{Z, Z}\left(\xi e^{i \omega_{1} t}\right)+D_{-Z, Z}\left(\xi e^{i \omega_{1} t}\right)\right. \\
& \left.+D_{Z,-Z}\left(\xi e^{i \omega_{1} t}\right)+D_{-Z,-Z}\left(\xi e^{i \omega_{1} t}\right)\right] \\
{ }_{o}\left\langle Z\left|e^{i \hat{\theta}(t)}\right| Z\right\rangle_{o}= & e^{i \omega_{0} t}\left[D_{Z, Z}\left(\xi e^{i \omega_{1} t}\right)-D_{-Z, Z}\left(\xi e^{i \omega_{1} t}\right)\right. \\
& \left.-D_{Z,-Z}\left(\xi e^{i \omega_{1} t}\right)+D_{-Z,-Z}\left(\xi e^{i \omega_{1} t}\right)\right]
\end{aligned}
$$


where the matrix elements of the displacement operator are given by Eqs. (14) and (15).

From Eqs. (33) and (34) we find that

$$
\begin{aligned}
& { }_{e}\left\langle Z\left|\left(\begin{array}{c}
\sin \hat{\theta} \\
\cos \theta
\end{array}\right)\right| Z\right\rangle_{e}=2 A_{+}(|Z|) e^{-\frac{1}{2} \xi^{2}}\left[\cos 2 u+e^{-2|Z|^{2}} \cosh 2 v\right]\left(\begin{array}{c}
\sin \omega_{0} t \\
\cos \omega_{0} t
\end{array}\right) \\
& { }_{o}\left\langle Z\left|\left(\begin{array}{c}
\sin \hat{\theta} \\
\cos \theta
\end{array}\right)\right| Z\right\rangle_{o}=2 A_{-}(|Z|) e^{-\frac{1}{2} \xi^{2}}\left[\cos 2 u-e^{-2|Z|^{2}} \cosh 2 v\right]\left(\begin{array}{c}
\sin \omega_{0} t \\
\cos \omega_{0} t
\end{array}\right)
\end{aligned}
$$

Then, we obtain the fluctuations of the supercurrent in the MJJ for even and odd CS

$$
\begin{aligned}
\left\langle(\Delta \sin \hat{\theta})^{2}\right\rangle_{e} & =\frac{1}{2}-B_{+} \cos 2 \omega_{0} t-C_{+}^{2} \sin ^{2} \omega_{0} t \\
\left\langle(\Delta \sin \hat{\theta})^{2}\right\rangle_{o} & =\frac{1}{2}-B_{-} \cos 2 \omega_{0} t-C_{-}^{2} \sin ^{2} \omega_{0} t
\end{aligned}
$$

where

$$
\begin{aligned}
& B_{ \pm}=A_{ \pm}(|Z|) e^{-2 \xi^{2}}\left(\cos 4 u \pm e^{-2|Z|^{2}} \cosh 4 v\right) \\
& C_{ \pm}=2 A_{ \pm}(|Z|) e^{-\frac{1}{2} \xi^{2}}\left(\cos 2 u \pm e^{-2|Z|^{2}} \cosh 2 v\right) .
\end{aligned}
$$

On substituting Eqs. (35)-(38) into Eq. (32), we find the squeezing degree of the supercurrents with respect to even and odd CS, respectively

$$
\begin{aligned}
& S_{I}^{e}(t)=\frac{1-2 B_{+} \cos 2 \omega_{0} t-2 C_{+}^{2} \sin ^{2} \omega_{0} t}{2 e\left|C_{+} \cos \omega_{0} t\right|}-1, \\
& S_{I}^{o}(t)=\frac{1-2 B_{-} \cos 2 \omega_{0} t-2 C_{-}^{2} \sin ^{2} \omega_{0} t}{2 e\left|C_{-} \cos \omega_{0} t\right|}-1
\end{aligned}
$$

which indicate that when $t \neq \frac{(2 n-1) \pi}{2 \omega_{0}}(n=1,2, \ldots)$ and

$$
1-2 B_{ \pm} \cos 2 \omega_{0} t-2 C_{ \pm} \sin ^{2} \omega_{0} t<2 e\left|C_{ \pm} \cos \omega_{0} t\right|
$$

we have $S_{I}^{e}(t)<0$ and $S_{I}^{o}(t)<0$ which means that the supercurrent exhibits squeezing. However, when $t=\frac{(2 n-1) \pi}{2 \omega_{0}}(n=1,2, \ldots)$, we have $S_{I}^{e}(t)=S_{I}^{o}(t)=+\infty$. From the uncertainty relation (27) and Eq. (31) we know that

$$
\left\langle(\Delta \hat{I})^{2}\right\rangle\left\langle(\Delta \hat{q})^{2}\right\rangle \geq 0
$$


which means that no squeezing of the supercurrent occurs under this circumstance.

We plot the time evolution of squeezing of the supercurrent in the MJJ when $\omega_{0}=2 N \omega_{1}$ for even and odd CS cases in Fig.1 and Fig.2. As can be seen from Fig.1, in the case of even CS, the squeezing is weakened with the increase of the value of $|Z|$ for a given $N$. Nevertheless, the case of odd CS is contrary to the even CS case. From Fig.2 we see that the squeezing is strengthened with the increase of the value of $|Z|$ for a given $N$, although there does not exist squeezing of the supercurrent when $Z$ is very small. It is noted that the singular points of the squeezing parameters appear periodically with the period $\frac{\pi}{\omega_{0}}$ which depends only on the external classical voltage bias, the number of the singular points increases with the increasing of $N$. Therefore, the angular frequency of the nonclassical EMF does not affect periodicity of the supercurrent squeezing. Comparing Fig.1 with Fig.2, we find that the squeezing curve of the odd CS approaches to that of the even CS with the increasing of $|Z|$ for a given $N$.

Since even and odd CS are nonclassical states while the Glauber CS are classical states, their effects on the squeezing of the supercurrent in the MJJ are quite different. To see this, we write out the squeezing degree of the supercurrent for Glauber CS,

$$
S_{I}(t)=\frac{\left(e^{\frac{1}{2} \xi^{2}}-e^{-\frac{1}{2} \xi^{2}}\right)\left\{1+e^{-\xi^{2}} \cos \left[4 \xi|Z| \sin \left(\omega_{1} t-\varphi\right)+2 \omega_{0} t\right]\right\}}{2 e\left|\cos \left[2 \xi|Z| \sin \left(\omega_{1}-\varphi\right)+\omega_{0} t\right]\right|}-1
$$

which indicates that when $2 \xi|Z| \sin \left(\omega_{1} t-\varphi\right)+\omega_{0} t \neq(2 n-1) \pi / 2$ and $\left(e^{\frac{1}{2} \xi^{2}}-e^{-\frac{1}{2} \xi^{2}}\right)$ $\left\{1+e^{-\xi^{2}} \cos \left[4 \xi|Z| \sin \left(\omega_{1} t-\varphi\right)+2 \omega_{0} t\right]\right\}<2 e\left|\cos \left[2 \xi|Z| \sin \left(\omega_{1}-\varphi\right)+\omega_{0} t\right]\right|$ the supercurrent exhibits squeezing (i.e., $\left.S_{I}(t)<0\right)$. When $2 \xi|Z| \sin \left(\omega_{1}-\varphi\right)+\omega_{0} t=$ $\frac{(2 n-1) \pi}{2}(n=1,2, \ldots)$, the squeezing disappears due to $S_{I}(t)=+\infty$ 
FIG.1: Time evolution of squeezing of the supercurrent in the MJJ when the external nonclassical EMF is in even CS for $\varphi=\frac{\pi}{2}$ and

(a) $|Z|=0.5, N=5$; (b) $|Z|=1, N=5$; (c) $|Z|=0.5 N=1$; (d) $|Z|=0.1 N=1$. 
FIG.2: Time evolution of squeezing of the supercurrent in the MJJ when the external nonclassical EMF is in odd CS for $\varphi=\frac{\pi}{2}$ and

(a) $|Z|=0.5, N=5$; (b) $|Z|=1, N=5$; (c) $|Z|=0.5 N=1$; (d) $|Z|=0.1 N=1$. 
FIG.3: Time evolution of squeezing of the supercurrent in the MJJ when the external quantum EMF is in the Glauber CS for $\varphi=\frac{\pi}{2}$ and

(a) $|Z|=10, N=5$; (b) $|Z|=10, N=1 ;$ (c) $|Z|=1 N=1$.

In Fig.3, we plot the time evolution of the squeezing of the supercurrent in one period in the presence of the Glauber coherent-state EMF when the step condition is satisfied, i.e., $\omega_{0}=N \omega_{1}$. Obviously, the squeezing of this case is quite different from that of even and odd CS. From (a) and (b) in Fig.3, we can see that for a given $|Z|$, 
oscillations of $S_{I}(t)$ are strenghtened during the preceding half period. From (b) and (c) we can see that for a given value of $N$, the oscillatary behaviors are weakened and the number of the singular points in every period decreases with the decreasing of $|Z|$.

\section{Quantum coherences of supercurrent in the MJJ in the presence of quantum EMF}

In this section we consider another important aspect of statistical properties of the supercurrent in the MJJ, that is its quantum coherences which describe the degree of quantum phase correlation in the supercurrent. The degree of the second-order quantum coherence can be obtained by evaluating the second-order correlation function of the supercurrent at two different spacetime points. If purely temporal correlations are of interest, the relevant correlation function in the normalized form can be defined by

$$
g^{(2)}(t, t+\tau)=\frac{\langle: \hat{I}(t) \hat{I}(t+\tau):\rangle}{\langle\hat{I}(t)\rangle\langle\hat{I}(t+\tau)\rangle}
$$

where the bracket " \langle\rangle " denotes taking expectation values over states of the external quantum EMF, and ": :" stands for normal ordering of quantum mechanical operators. Following considerations in quantum optics, We are interested in behaviors of the second-order correlation function (44) at $\tau=0$. Then, from Eq. (44) we get

$$
g^{(2)}(t) \equiv g^{(2)}(t, t)=\frac{\left\langle: \hat{I}^{2}(t):\right\rangle}{\langle\hat{I}(t)\rangle^{2}}
$$

Substituting the expression of the supercurrent operator (5) into the above equation and making use of the formula

$$
: D\left(2 \xi e^{i \omega_{1} t}\right):=e^{2 \xi^{2}} D\left(2 \xi e^{i \omega_{1} t}\right)
$$

we find the general expression of the second-order correlation function in the following form

$$
g^{(2)}(t)=\frac{1-e^{2 \xi^{2}} \operatorname{Re}\left\langle e^{i 2 \theta}\right\rangle}{2\langle\sin \theta\rangle^{2}}
$$


For even and odd CS, we find that

$$
g_{e}^{(2)}=\frac{1-2 B_{+} \cos 2 \omega_{0} t}{2 C_{+} \sin ^{2} \omega_{0} t}, \quad g_{o}^{(2)}=\frac{1-2 B_{-} \cos 2 \omega_{0} t}{2 C_{-} \sin ^{2} \omega_{0} t}
$$

where $B_{ \pm}$and $C_{ \pm}$are given by Eq. (40).

From Eq. (48), it can be seen that when $t=\frac{n \pi}{\omega_{0}}(n=0,1,2, \ldots)$ we have $g_{e}^{(2)}(t)=$ $g_{o}^{(2)}(t)=+\infty$ which indicates that quantum coherences vanish since the mean value of the supercurrent is zero at these points.

FIG.4: Time evolution of quantum coherence of the supercurrent when the external nonclassical EMF is in even CS for $\varphi=\frac{\pi}{2}$

$$
\text { and (a) }|Z|=0.5, N=1 \text {; (b) }|Z|=0.5, N=2 \text {. }
$$

In Fig.4 and Fig.5 we plot the time evolution of quantum coherences of the supercurrent in the MJJ when $\omega_{0}=2 N \omega_{1}$ for even CS and odd CS, respectively. As can be seen from these figures that when the external nonclassical EMF is in even and odd 
CS, the supercurrent in the MJJ exhibits similar quantum coherences during the time evolution.

FIG.5: Time evolution of quantum coherence of the supercurrent when the external nonclassical EMF is in odd CS for $\varphi=\frac{\pi}{2}$

$$
\text { and (a) }|Z|=0.5, N=1 \text {; (b) }|Z|=0.5, N=2 \text {. }
$$

It is worthwhile mentioning that when the external quantum EMF is in Glauber $\mathrm{CS}$, the time evolution of quantum coherences of the supercurrent in the MJJ is quite different from that of the even and odd CS cases. For the case of the Glauber CS, it is quite easy to find the second order correlation function to be $g^{(2)}(t)=e^{\xi} \doteq 1$ which means that the quantum coherence of the supercurrent in the presence of the coherent-state EMF does not change during the time evolution. Therefore, nonclassical states and classical states of the external quantum EMF lead to quite different time evolution of quantum coherences of the supercurrent in the MJJ, in this sense, the MJJ can distinguish the external nonclassical light fields and classical light fields. 


\section{Concluding remarks}

We have studied some properties of the supercurrent in the MJJ in the presence of nonclassical light fields, the CVSS, squeezing effect and quantum coherences. In particular, we have investigated in detail these properties when the external quantum EMF is in even and odd CS. We have found that the CVSS of the MJJ depends strongly on the off-diagonal elements of the density matrix which describes the external quantum EMF. We have shown that the supercurrent in the MJJ exhibits both squeezing and quantum coherences. However, when the CVSS of the MJJ is satisfied, squeezing and quantum coherences vanish periodically due to the periodical appearance of singular points of the squeezing parameter and the second-order correlation function of the supercurrent. we have also shown that the MJJ can feel the difference not only between classical light fields and nonclassical light fields but also betwwen different nonclassical light fields. This indicates that the MJJ are very sensitive devices that can respond to the quantum nature of external nonclassical light fields. Therefore, the MJJ is a sensitive detector to nonclassical light fields. We believe that the results obtained in this paper could be useful in the study of the controlling of Josephson tunnelling and the detection of nonclassical light fields in quantum optics.

The research was supported in part by the National Nature Science Foundation of China. 


\section{References}

[1] G. Schon and A. D. Zaikin, Phys. Rep. 198, 237(1990).

[2] D. V. Averin and K. K. Likharev, J. Low Temp. Phys. 62, 345(1986).

[3] A. Widom, G. Megaloudis, T. D. Clark and R. J. Prance, J. Phys. A15, $1561(1982)$.

[4] A. Widom, G. Megaloudis, T. D. Clark, J. E. Mutton, R. J. Prance and H. Prance, J. Low Temp. Phys. 57, 651(1984).

[5] F. W. J. Hekking, L. I. Glazman, K. A. Matreev and R. I. Shekhter, Phys. Rev. Lett. 70, 4138(1993).

[6] G. Ingold and H. Grabert, Phys. Rev. B50, 395(1994); R. Bauerrschmitt, J. Siewert, Y. V. Nazarov and A. A. Odintsov, Phys. Rev. B49, 4076(1994).

[7] K. K. Likharev and A. B. Zorin, "Proceedings of the International Conference on Low Temperature Physics -LT-17", edited by U. Eckern, A. Schmid, W. Weber and H. Wühl (Elsevier, Amsterdam, 1984), p.1153.

[8] K. K. Likharev and A. B. Zorin, J. Low Temp. Phys. 59, 347(1985).

[9] D. V. Averin, A. B. Zorin and K. K. Likharev, Sov. Phys. -JETP 61, 407(1985).

[10] M. Büttiker, Phys. Rev. B36, 3548(1987).

[11] A. O. Caldeira and A. J. Leggett, Phys. Rev. Lett. 46, 211(1981); Ann. Phys.(NY) 149, 374(1983).

[12] R. F. Voss and R. A. Webb, Phys. Rev. Lett. 47, 265(1981); S. Washburn, R. A. Webb, R. F. Voss and S. M. Fairs, Phys. Rev. Lett. 54, 2712(1985). 
[13] L. D. Jackel, J. P. Gordon, E. L. Hu, R. E. Howard, L. A. Fetter, D. M. Tennant, R. W. Epworth and J. Kurkijärvi, Phys. Rev. Lett. 47, 697(1981).

[14] M.H. Devoret, J.M. Martinis and J. Clarke, Phys. Rev. Lett. 55, 1908(1985).

[15] D. B. Schartz, B. Sen, C. N. Archie and J. E. Lukens, Phys. Rev. Lett. 55, $1547(1985)$

[16] S. Shapiro, Phys. Rev. Lett. 11, 80(1963).

[17] R. Loudon and P. L. Knight, J. Mod. Opt. 34, 709(1987); M. C. Teich and B. E. A. Saleh, Quantum Opt. 1, 153(1989).

[18] J. Perina, "Quantum statistics of linear and nonlinear optical phenomena" (Reidel, Dordrecht, 1984), p.78.

[19] V. Bužek, A. Vidiella-Barranco and P. L. Knight, Phys. Rev. A45, 6570(1992).

[20] N. A. Ansari, L. D. Fiore, M. A. Man'ko, V. I. Man'ko, S. Solimeno and F. Zaccarria, Phys. Rev. A49, 2151(1994); A. Vidiella-Barranco, H. Moya-Cessa and V. Bužek, J. Mod. Opt. 39, 1441(1992).

[21] J. Gea-Banacloche, Phys. Rev. A44, 5913(1991); V. Bužek, H. Moya-Cessa, P.L. Knight, and S. J. D. Phoenix, Phys. Rev. A45, 8190(1992).

[22] M. Brune, S. Haroche, J. M. Raimond, L. Davidovich and N. Zagury, Phys. Rev. A45, 5193(1992).

[23] C. C. Gerry, Opt. Commun. 91, 247(1992); C. C. Gerry and E. E. Hach III, Phys. Lett. A174, 185(1993).

[24] F. B. Wang and L. M. Kuang, Phys. Lett. A169, 225(1992); L. M. Kuang and F. B. Wang, Phys. Lett. A173, 221(1993). 
[25] D.F. Walls, Nature, 306, 141(1983); P. Tombesi and E. R. Pike, "Squeezed and non-classical light" (Plenum, New York, 1989).

[26] F. B. Wang and L. M. Kuang, J. Phys. A26, 293(1993); Y. Xia and G. Guo, Phys. Lett. A136, 281(1989).

[27] A. Vourdas, Phys. Rev. B49,12040(1994).

[28] For a review on the subject see H. J. Kimble and D. F. Walls, J. Opt. Soc. Am. B4(1987)(special issue on squeezed states of the electromagnetic field); L. M. Kuang, F. B. Wang and Y. G. Zhuo, J. Mod. Opt. 41, 1307(1994).

[29] P. Carruthers and M. M. Nieto, Phys. Rev. Lett. 14, 387(1965).

[30] L. M. Kuang and X. Chen, Phys. Rev. A50 4228(1994); Phys. Lett. A186, 8(1994).

[31] A. Perelomov, "Generalized coherent states and their applications" (SpringerVerlag, Berlin, 1986).

[32] J. M. Radcliffe, J. Phys.(Paris) A4, 313(1971);

F. T. Arecchi, E. Courtens, R. Gilmore and H. Thomas, Phys. Rev. A6, 2211 (1972).

[33] K. Wódkiewicz and J.H. Eberly, J. Opt. Soc. Am. B2, 458(1985). 